\title{
Collective Lipid Bilayer Dynamics Excited by Surface Acoustic Waves
}

\author{
T. Reusch, ${ }^{1}$ F. J. R. Schülein, ${ }^{3,4}$ J. D. Nicolas, ${ }^{1}$ M. Osterhoff, ${ }^{1}$ A. Beerlink, ${ }^{5}$ \\ H. J. Krenner, ${ }^{3,4}$ M. Müller, ${ }^{2}$ A. Wixforth, ${ }^{3,4}$ and T. Salditt ${ }^{1, *}$ \\ ${ }^{1}$ Institut für Röntgenphysik, University of Göttingen, Friedrich-Hund-Platz 1, 37077 Göttingen, Germany \\ ${ }^{2}$ Institut für Theoretische Physik, Georg-August-Universität Göttingen, Friedrich-Hund-Platz 1, 37077 Göttingen, Germany \\ ${ }^{3}$ Lehrstuhl für Experimentalphysik I, Universität Augsburg, Universitätsstr. 1, 86159 Augsburg, Germany \\ ${ }^{4}$ Nanosystems Initiative Munich, Schellingstrasse 4, 80799 Munich, Germany \\ ${ }^{5}$ Deutsches Elektronen-Synchrotron, Notkestrasse 85, 22605 Hamburg, Germany
}

(Received 20 March 2014; published 9 September 2014)

\begin{abstract}
We use standing surface acoustic waves to induce coherent phonons in model lipid multilayers deposited on a piezoelectric surface. Probing the structure by phase-controlled stroboscopic x-ray pulses we find that the internal lipid bilayer electron density profile oscillates in response to the externally driven motion of the lipid film. The structural response to the well-controlled motion is a strong indication that bilayer structure and membrane fluctuations are intrinsically coupled, even though these structural changes are averaged out in equilibrium and time integrating measurements. Here the effects are revealed by a timing scheme with temporal resolution on the picosecond scale in combination with the sub-nm spatial resolution, enabled by high brilliance synchrotron $\mathrm{x}$-ray reflectivity.
\end{abstract}

The equilibrium properties of lipid membranes are well understood [1,2]. In particular, the molecular structure and dynamics of the lipid bilayers as model systems for biological membranes can be studied at high spatial and temporal resolution, for example, by inelastic neutron scattering, revealing a rich spectrum of thermal shape fluctuations and the characteristic dispersion relations $[3,4]$. However, scattering techniques have to date been limited to large ensembles probed in thermal equilibrium. Generically, however, biological membranes are out of equilibrium. The membrane is subjected to external forces, i.e., due to osmotic pressure differences, the coupling to an active cytoskeleton, or to membrane proteins such as ion channels or pumps. These effects change structural, dynamical, and mechanical properties on a fundamental level. Nerve pulse propagation is one important example where the interplay between nonequilibrium forces and pressure has been postulated to couple to structural changes and phase transitions in membranes [5], which would have considerable implications for physiology [6]. In order to gain further insight into these matters, the lack of experimental probes with sufficiently high spatial and temporal resolution under controlled out-of-equilibrium parameters has presented a tremendous challenge.

Here we use time resolved $\mathrm{x}$-ray diffraction to study outof-equilibrium structural dynamics of lipid membranes, using surface acoustic waves (SAWs) for well controlled

Published by the American Physical Society under the terms of the Creative Commons Attribution 3.0 License. Further distribution of this work must maintain attribution to the author(s) and the published article's title, journal citation, and DOI. excitations [7]. SAWs have previously been shown to induce phase segregation in multicomponent lipid bilayers as probed by fluorescence microscopy $[8,9]$, and to some extent the electroacoustic fields can mimick the effects of external forces which act on biomolecular assemblies or membranes in a biological environment. Up to now, however, the limited experimental resolution has impeded clear-cut explanations of many such phenomena, since the spatial and temporal scales of the molecular dynamics have not been accessed. Combining structural resolution on the sub-nm scale with temporal resolution on the picosecond scale, we are now able to directly monitor periodic changes in the electron density profile $\Delta \rho(z)$ of the lipid bilayers, induced by the coherent phonon emitted by the SAW device into the stack of multilamellar membranes deposited on top of the piezoelectric surface. From the observations we infer that the lipid bilayers are not just undergoing simple harmonic translations during the SAW cycle, but that the macroscopic acoustic wave couples to the arrangement and conformations of lipids and water within a periodic unit of the membrane stack.

In this work, SAWs are generated on piezoelectric lithium niobate $\mathrm{LiNbO}_{3}$ substrates by a metallic comblike finger structure [10], the so-called interdigital transducer (IDT); see Fig. 1. These IDTs are excited by a radio frequency (rf) voltage at the design frequency $f_{\mathrm{SAW}}=v_{\mathrm{SAW}} / L_{\mathrm{SAW}}$, given by the SAW velocity $v_{\mathrm{SAW}} \approx 3670 \mathrm{~m} / \mathrm{s}$ and the finger distance $L_{\text {SAW }}$ (typically $10-100 \mu \mathrm{m}$ ), leading to SAW propagation along the substrate surface. In the present experiment the acoustically active region (sound path) was $1 \mathrm{~mm}$ wide and was bounded by a second identical IDT at a distance of $8 \mathrm{~mm}$. Standing SAWs were generated 


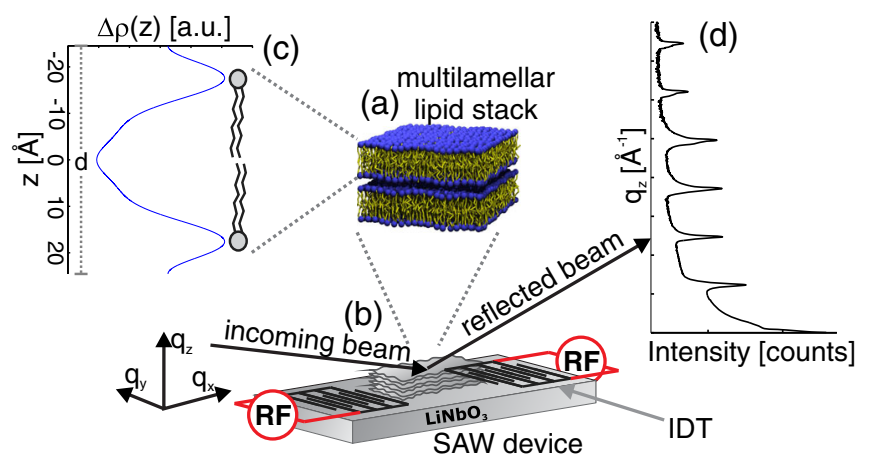

FIG. 1 (color online). (a) Multilamellar stacks of lipid bilayers deposited on the piezoelectric $\mathrm{LiNbO}_{3}$ substrate of a SAW device. (b) By application of a rf signal to the IDTs, standing waves or propagating SAW pulses are induced. The ultrafast response of (c) the averaged bilayer electron density profile $\Delta \rho(z)$ is measured by (d) time resolved (phase locked) x-ray reflectivity experiments.

by phase and frequency matched excitation of both opposing IDTs. The finger periodicity was $L_{\mathrm{SAW}}=100 \mu \mathrm{m}$, corresponding to a fundamental design frequency of $f_{\text {SAW }}^{1}=$ 36.7 MHz. Resulting from the propagation direction or cut angle used, only Rayleigh waves (elliptic motion in the sagittal plane) are excited [11]. In addition to the mechanical component of the SAW, the piezoelectricity of the substrate material induces an electric potential $\Phi$ which is linked to the deformation of the crystal. Both the lattice deformation and $\Phi$ decay exponentially into the substrate, while the electric potential also decays exponentially into the vacuum above the surface. As long as the distance from the surface is small compared to the wavelength $L_{\mathrm{SAW}}$, the electric potential $\Phi$ and, thus, the electric field $E_{z}=-\partial \Phi / \partial z \approx 10^{5} \mathrm{~V} / \mathrm{m}$ will, in general, affect a thin film on the surface, as detailed in the Supplemental Material [12].

The pulsed nature of synchrotron radiation with x-ray pulse lengths on the order of $50 \mathrm{ps}$ and pulse frequencies of several $\mathrm{MHz}$ enables a broad range of time-resolved experiments including inelastic scattering such as nuclear resonant scattering, $x$-ray spectroscopy (EXAFS, NEXAFS fluorescence [17]), or diffraction [18-21]. Phase locked stroboscopic diffraction experiments can be performed by matching the frequency $f_{\mathrm{SAW}}=n f_{b}$ of standing SAWs to any integral multiple $n$ of the synchrotron bunch frequency $f_{b}$ [11]. Subsequent $\mathrm{x}$-ray pulses thus probe the standing SAW at a fixed phase $\phi$, resulting in stroboscopic snapshots of the SAW induced dynamics [22]. A variation of $\phi$ allows sampling of the structural changes at a high number of time points, with the temporal resolution given by the $\mathrm{x}$-ray pulse length of $\approx 50 \mathrm{ps}$ (and negligible electronic jitter). Oriented multilamellar stacks of $N \approx 1300$ 1,2-Dioleoyl-sn-glycero3-phosphatidylcholine (DOPC) bilayers have been deposited on carefully cleaned $\mathrm{LiNbO}_{3}$ substrates, following the procedures described in [23]. Individual bilayers are highly aligned with respect to the substrate surface with a well defined interbilayer periodicity $d$ leading to sharp Bragg reflections at $q_{z}(h)=h(2 \pi / d)$. Time resolved reflectivity experiments have been performed at a x-ray energy of $18 \mathrm{keV}$ at the high resolution diffraction beam line P08, PETRA III, DESY [24] using the PETRA III 40-bunch mode $\left(f_{b} \approx 5.2 \mathrm{MHz}\right)$, yielding $I(h, \phi)$ for each multilamellar reflection $h$ as a function of phase shift $\phi$ between the standing wave and the subsequent synchrotron bunches. Note, that a phase shift of $\phi$ is equivalent to an effective time delay $t=(\phi / 360) \cdot\left(1 / f_{\mathrm{SAW}}\right)$. A SAW frequency of $f_{\mathrm{SAW}}=7 f_{b}=36.4 \mathrm{MHz}$ (closely matched to the design frequency of the IDTs) has been chosen, the SAW period is therefore $\approx 27.5 \mathrm{~ns}$. An electric power of $P_{\mathrm{SAW}}=21 \mathrm{dBm}$ was applied to each IDT, as detailed in [11], resulting in a SAW amplitude $a$ of about $3 \AA \leq a \leq 1 \mathrm{~nm}$. Lamellar Bragg reflections have been recorded in the fluid phase with the sample at room temperature and hydrated from vapor in a home-built sample chamber with Mylar windows and electric feedthrough. Measurements covered up to the sixth lamellar orders $(1 \leq h \leq 6)$. Four exemplary intensity traces $I(1 \leq h \leq 4, \phi)$ are depicted in Fig. 2. Three successive identical measurements were performed in each case, yielding perfectly reproducible results. The averaged $I(h, \phi)$ were modeled by an eighth-order Fourier series $\left[I(h, \phi)=a_{0}(h)+\sum_{i=1}^{8} a_{i}(h) \cos (\phi)+b_{i}(h) \sin (\phi)\right] \quad$ in order to extract the leading temporal Fourier components $a_{i}, b_{i}$ for further analysis.

The measured $I(h, \phi)$ curves exhibit three particularly striking features: (i) The $I(h, \phi)$ curves corresponding to different reflection orders $h$ are out of phase; see $h=1$ and $h=2$ traces in Fig. 2. (ii) The functional form of $I(h, \phi)$ does not match the sinusoidal line shape of the exciting SAW. Notably, $I(2, \phi)$ exhibits three intensity maxima for

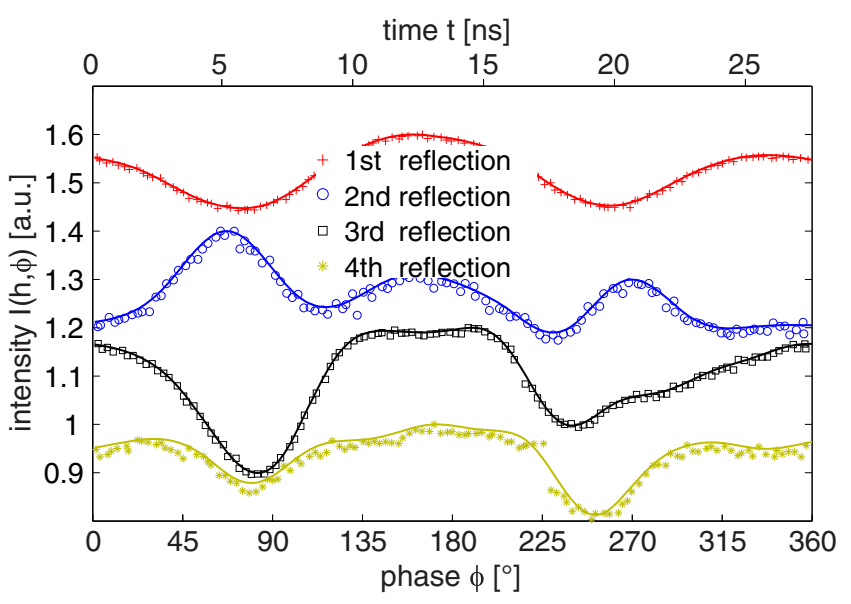

FIG. 2 (color online). The integrated intensities $I(h, \phi)$ of the first four diffraction orders $h$ of the multilamellar lipid stack plotted as a function of relative phase $\phi$. Individual intensity traces $I(h, \phi)$ are out of phase, pointing to a complex interaction between the SAW and the lipid multilayer structure. The plots have been shifted for clarity. 
one period of the SAW. (iii) $I(h, \phi)$ is not (completely) symmetric for phase shifts of $\Delta \phi=180^{\circ}$ but is for phase shifts of $\Delta \phi=360^{\circ}$. These observations are corroborated by various complementary experiments, including different lipid systems, SAW devices, and frequencies during two beam times; see [12]. The experimental findings can, in principle, be attributed to either (1) a phase dependent mean-square fluctuation amplitude $\sigma$ (i.e., SAW induced disorder), (2) structure-factor effects corresponding to, e.g., changes of the inter- and intrabilayer correlation functions, or (3) form-factor effects reflecting changes on the scale of individual bilayers. Given observations (i) and (ii) we can discard a SAW induced Debye-Waller factor, i.e., a phase dependent $\sigma$, in the data interpretation as associated changes in $I(h, \phi)$ would necessarily be in phase for different $h$. Further, time resolved reflectivity and rocking measurements have been performed in order to disentangle form-factor effects from structure-factor effects. The $\phi$ dependent intensity oscillations are not accompanied by oscillations of the peak position, width, or line shape [12]. We therefore conclude that the observed fast dynamics are related to bilayer form factor variations, rather than a time dependent structure factor of the multilamellar stack. Thus, the observed specular intensity traces $I(h, \phi)$ cannot be explained by SAW induced changes in the correlation functions. They can only be explained by bilayer formfactor changes and, hence, structural changes on the level of individual bilayers. Therefore, the next step is to reconstruct the electron density profiles $\Delta \rho(z, \phi)$ (relative to the mean density) from the measured $I(h, \phi)$ for each phase $\phi$, see [12] for details. The required phase factors $\nu_{h} \in$ $[-1,+1]$ have been taken from reference measurements without SAW excitation [25] and are set constant over the whole time range as changes of $\nu_{h}$ would be connected to an unobserved zero crossing of the corresponding Bragg intensity. Furthermore absolute values of $F\left[q_{z}(h)\right]$ have been calculated by scaling the measured integrated peak intensities $I(h, \phi)$ to reference reflectivity experiments under matching ambient conditions, so that only the measured relative variations of $I(h, \phi)$ enter the analysis. By this procedure, the comparatively poor sample quality of lipid multilayers on $\mathrm{LiNbO}_{3}$ substrates did not affect the data analysis. The data quality was improved in a subsequent beam time [12]. Note also that due to uncertainties related to cable lengths and the electromechanical interaction at the IDTs, the true phase-zero $\phi_{0}$ do not necessarily correspond to the nominal $\phi=0^{\circ}$ in Fig. 2 and Fig. 3, but have to be identified from the measured data [11]. Figure 3(a) shows the resulting electron density profiles $\Delta \rho(z, \phi)$. During the SAW period, pronounced changes of $\Delta \rho(z, \phi)$ in the headgroup region $(z \approx \pm 17 \AA)$ as well as in the hydrophobic core $(z \approx 0 \AA)$ are observed, accompanied by a variation of the bilayer thickness. To further quantify these effects, characteristic bilayer parameters (bilayer thickness $d_{h h}$, and headgroup width $d_{h w}$ ) are
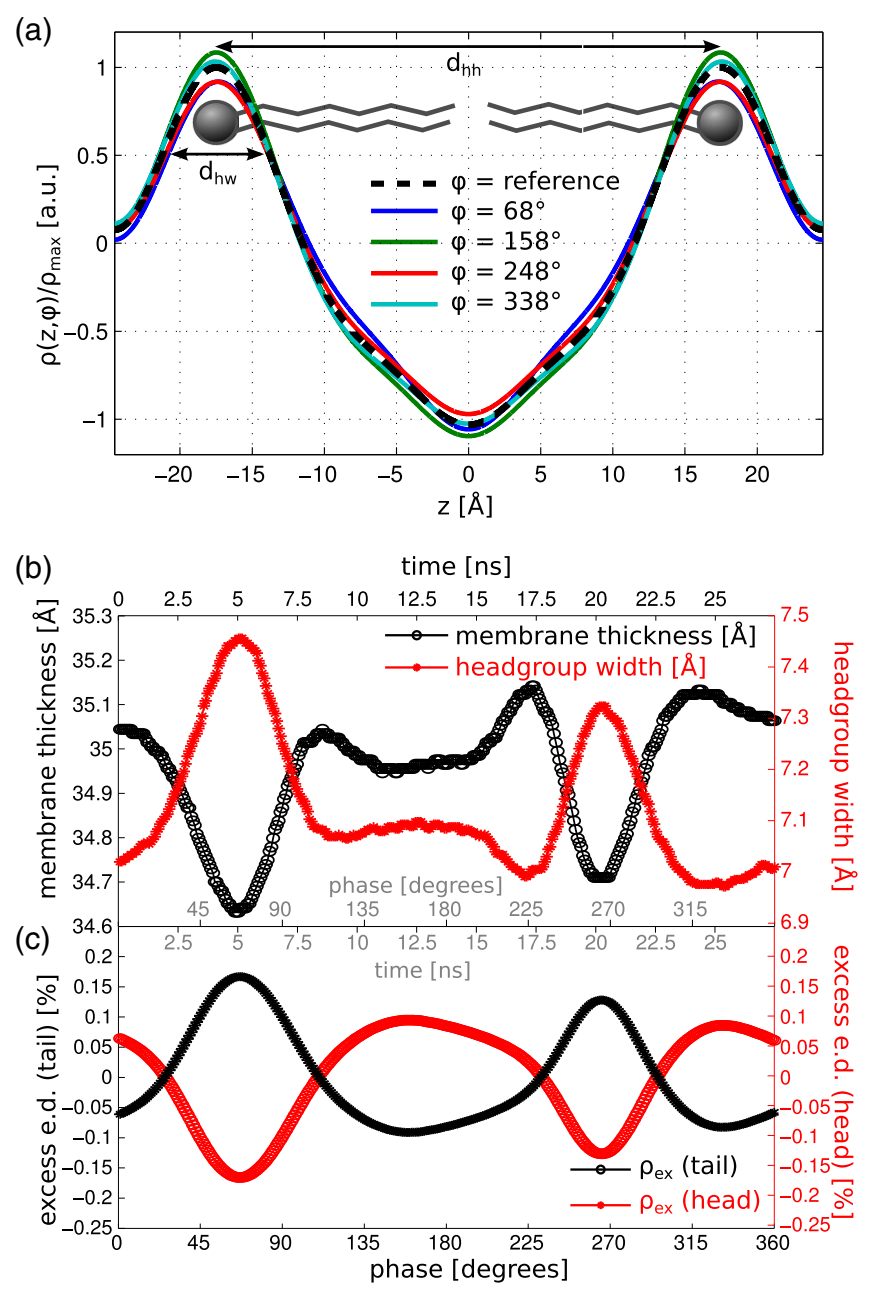

FIG. 3 (color online). (a) Structural dynamics of the lipid bilayer in response to the acoustic excitation by the SAW, as quantified by the time resolved electron density profile, shown for selected phase angles $\phi$ along with the equilibrium reference profile (dashed line). Pronounced variations of the resulting relative electron density $\Delta \rho(z, \phi)$ are observed. The density variations are accompanied by a variation of membrane thickness. (b) Characteristic bilayer parameters (membrane thickness and headgroup width) extracted from $\Delta \rho(z, \phi)$. The maxima in the headgroup width $d_{h w}$ are accompanied by minima of the membrane thickness $d_{h h}$, and (c) a reduction of the electron density in the headgroup region (negative excess density) compensated for by a density increase in the tail region (positive excess density).

extracted from the $\Delta \rho(z, \phi)$; see Fig. 3(b) for the corresponding temporal evolution. Here, $d_{h h}$ is defined as the headgroup-headgroup distance between the maxima of $\Delta \rho(z, \phi)$, and $d_{h w}$ as the FWHM of the headgroup maximum [26]. While the periodicity $d$ remains constant throughout the measurement, the maximal membrane thickness change $\delta \approx 0.2 \AA$ corresponds to a relative amplitude $\delta / a \simeq 2 \%$ with respect to the amplitude of the SAW excitation [11]. Resulting from lateral averages, the density in each plane contains contributions of both water 
and protruding lipid headgroup moieties. Figure 3(c) quantifies the associated oscillations in the excess electron densities in the headgroup and lipid tail moieties, integrated between the zero crossings. Since zero denotes the average density over one multilamellar period (bilayer plus water), the sum of the excess densities is always zero. The extrema of the oscillation cycle, at $\phi \simeq 68^{\circ}$ and $\phi \simeq 248^{\circ}$, associate the local maxima in $d_{h w}$ and minima in $d_{h h}$ with negative excess density in the headgroups and positive excess density in the tail region, i.e., corresponding to a density profile which is less accentuated than that of the equilibrium fluid phase. The opposite is true for $\phi \simeq 156^{\circ}$ and $\phi \simeq 338^{\circ}$, where the bilayer profile is more accentuated, i.e., changes in the same direction as from the fluid to the gel phase. However, in contrast to equilibrium phase transitions, the apparent compression or expansion of $d_{h h}$ and the associated density changes must be brought about by a volume conserving redistribution of lipid moieties, since the compressibility of water is low and large scale mass transport is quenched. For example, changes in the inclination (tilt) angle of the lipid headgroups from its equilibrium value $\alpha \approx 60^{\circ}$ with respect to the surface normal [27] and redistribution of water could cause the observed effects.

Before further interpretation, let us first identify the primary driving force for these collective time dependent changes in the bilayer form factor. Two reference experiments have been carried out in order to disentangle electric field driven effects from mechanical effects. (i) Two identical parallel sound paths (Rayleigh waves, SAW frequency $f_{\mathrm{SAW}}^{1}=80 \mathrm{MHz}$ ) were prepared on the same SAW device. One path was covered by a $50 \mathrm{~nm}$ thin Au layer, effectively shielding the electric field [28]. Time resolved experiments on DOPC lipid multilayers were then carried out on both sound paths, with identical results. (ii) A pure shear wave was induced instead of Rayleigh waves reported above. Shear waves are characterized by pure in-plane displacements, no mechanical components perpendicular to the substrate surface are excited. The magnitude of electric field components $E_{z}$ perpendicular to the sample surface is, however, comparable to the case of Rayleigh waves [10]. In this case, no ultrafast effects have been observed. Therefore, we conclude that the observed periodic changes in bilayer structure are driven by the transverse component of the SAW. Further, based on the known dispersion relations in lipid stacks (undulation and baroclinic modes $[3,29,30]$ ) we can neglect flow and curvature effects in response to the acoustic excitation, see the Supplemental Material [12] for details, given the SAW wavelength $L=100 \mu \mathrm{m}$, frequency $f_{\text {SAW }}=36.4 \mathrm{MHz}$, SAW amplitude $a \leq 1 \mathrm{~nm}$, and the film thickness $L=6.5 \mu \mathrm{m}$.

With these simplifications and constraints in mind, we can consider as the simplest model a one-dimensional lattice with a unit cell composed of two slabs with thickness $d_{h h}(t)$ and $d_{w}$, representing the bilayer and water layer, subjected to harmonic acoustic excitation. In line with the experimental data, the bilayer periodicity $d=d_{w}+d_{h h}$ is kept constant. The acceleration pressure then induces periodic changes in the bilayer thickness $d_{h h}$, with maximum amplitude $\delta / a \simeq 2 \pi^{2} \rho d^{2} f_{\text {SAW }}^{2} / Y$ [12]. Here, $Y$ is an effective modulus for the degree of freedom associated with the observed membrane profile changes. While the $f_{\text {SAW }}^{2}$ dependence of the response amplitude is confirmed by a data set recorded at higher driving frequency [12], an unreasonably small modulus in the range $Y \simeq 10^{4}-$ $10^{5}\left(\mathrm{~N} / \mathrm{m}^{2}\right)$ is required to match the experimental values. Note that while moduli in this range have been determined from the voltage dependent capacitance of a BLM before [31], the Young's modulus describing uniaxial compression of a lipid bilayer is mostly cited to be 3 orders of magnitude higher [32]. We therefore conclude that the structural changes cannot be explained by the equilibrium compression or expansion of the bilayer. Instead, soft modes of the headgroup region, related, for example, to changes of the headgroup tilt angle, could well explain the observed effects, while preserving three-dimensional density and volume, which pose a constraint to the conformational changes in the absence of large scale flow [12]. The results show that collective structural changes on molecular scales can become relevant for soft matter systems such as lipid membranes, when excited acoustically at velocities and accelerations on the order of $1 \mathrm{~nm} / \mathrm{ns}$ and $1 \mathrm{~nm} /(\mathrm{ns})^{2}$, respectively. These oscillation periods, amplitudes, velocities, and accelerations could also occur in active membranes subject to rapidly changing forces, even if temporal and ensemble averaging would render detection or observation impossible (without synchronization). In fact, for phosphatidylcholines, an equilibrium amplitude of $3.7 \AA$ for the fluid phase thickness fluctuations, which has recently been reported from neutron spin echo spectroscopy [33], could be explained by similar collective soft modes, which would then couple to acoustic excitation.

In summary, the "structural bilayer movie" reconstructed from the reflectivity data shows thickness and density oscillations of the headgroup and lipid tail moieties, which are driven by the acoustic excitation (SAW). Further studies using the present approach should cover a broader range of experimental parameters (lipid composition, excitation, ambient conditions) and include more details of the structural dynamics, which could also play a role in physiological membrane processes.

We thank Dr. Oliver Seeck and Kathrin Pflaum for excellent support prior to, as well as during, the synchrotron experiment at P08, PETRA III, as well as Christina Bömer for help during the first beam time. The project has been funded by the German Research Foundation (Deutsche Forschungsgemeinschaft, DFG) through SFB 937 as well as through BMBF-project 05K13MG4. H. K. acknowledges support via the Emmy Noether Program (KR3790/2). 
*Corresponding author. tsaldit@gwdg.de

[1] R. Lipowsky and E. Sackmann, Structure and Dynamics of Membranes (Elsevier, New York, 1995).

[2] Lipid Bilayers: Structure and Interactions, edited by J. Katsaras and T. Gutberlet (Springer, New York, 2000).

[3] M. C. Rheinstädter, W. Häußler, and T. Salditt, Phys. Rev. Lett. 97, 048103 (2006).

[4] T. Salditt, M. Vogel, and W.Fenzl, Phys. Rev. Lett. 90, 178101 (2003).

[5] T. Heimburg and A. D. Jackson, Proc. Natl. Acad. Sci. U.S.A. 102, 9790 (2005).

[6] J. Griesbauer, S. Bössinger, A. Wixforth, and M. F. Schneider, Phys. Rev. Lett. 108, 198103 (2012).

[7] T. Frommelt, M. Kostur, M. Wenzel-Schäfer, P. Talkner, P. Hänggi, and A. Wixforth, Phys. Rev. Lett. 100, 034502 (2008).

[8] M. Hennig, M. Wolff, J. Neumann, A. Wixforth, M. F. Schneider, and J. O. Raedler, Langmuir 27, 14721 (2011).

[9] M. Hennig, J. Neumann, A. Wixforth, J. O. Radler, and M. F. Schneider, Lab Chip 9, 3050 (2009).

[10] H. Matthews, Surface Wave Filters (Wiley, New York, 1977), p. 521.

[11] T. Reusch, F. Schülein, C. Bömer, M. Osterhoff, A. Beerlink, H. J. Krenner, A. Wixforth, and T. Salditt, AIP Adv. 3, 072127 (2013).

[12] See Supplemental Material at http://link.aps.org/ supplemental/10.1103/PhysRevLett.113.118102 for complementary data sets, as well as further justifications of the conclusions presented here and a simplified model for the bilayer response to SAW excitation as well as a description of the solution to the piezoelectric wave equation in the case of Rayleigh modes, which includes Refs. [13-16].

[13] A. E. Blaurock, Biochimica et Biophysica Acta, 650, 167 (1982).

[14] J. Katsaras, Biochem. Cell Biol., 73, 209 (1995).

[15] J. Torbet and M.H. F. Wilkins, J. Theor. Biol., 62, 447 (1976).
[16] R. Ribotta, D. Salin, and G. Durand, Phys. Rev. Lett. 32, 6 (1974).

[17] L. X. Chen, W. J. Jäger, G. Jennings, D. Gosztola, D. J. Munkholm, and J. P. Hessler, Science 292, 262 (2001).

[18] M. Cammarata, M. Levantino, F. Schotte, P. A. Anfinrud, F. Ewald, J. Choi, A. Cupane, M. Wulff, and H. Ihee, Nat. Methods 5, 881 (2008).

[19] S. Techert, F. Schotte, and M. Wulff, Phys. Rev. Lett. 86, 2030 (2001).

[20] A. Plech, V. Kotaidis, M. Lorenc, and J. Boneberg, Nat. Phys. 2, 44 (2006).

[21] C. Rose-Petruck, R. Jimenez, T. Guo, A. Cavalleri, C. W. Siders, F. Rksi, J. A. Squier, B. C. Walker, K. R. Wilson, and C. P. J. Barty, Nature (London) 398, 310 (1999).

[22] W. Sauer, M. Streibl, T. H. Metzger, A. G. C. Haubrich, S. Manus, A. Wixforth, J. Peisl, A. Mazuelas, J. Hartwig, and J. Baruchel, Appl. Phys. Lett. 75, 1709 (1999).

[23] T. Salditt, J. Phys. Condens. Matter 17, R287 (2005).

[24] O. H. Seeck et al., J. Synchrotron Radiat. 19, 30 (2012).

[25] S. Aeffner, T. Reusch, B. Weinhausen, and T. Salditt, Proc. Natl. Acad. Sci. U.S.A. 109, E1609 (2012).

[26] J. F. Nagle and S. Tristram-Nagle, Biochim. et Biophys. Acta 1469, 159 (2000).

[27] R. A. Boeckmann, B. L. de Groot, S. Kakorin, E. Neumann, and H. Grubmueller, Biophys. J. 95, 1837 (2008).

[28] A. Wixforth, J. Scriba, M. Wassermeier, J. P. Kotthaus, G. Weimann, and W. Schlapp, Phys. Rev. B 40, 7874 (1989).

[29] C. L. Armstrong, W. Häußler, T. Seydel, J. Katsaras, and M. C. Rheinstädter, Soft Matter 10, 2600 (2014).

[30] H. Bary-Soroker and H. Diamant, Phys. Rev. E 76, 042401 (2007).

[31] J. M. Crowley, Biophys. J. 13, 711 (1973).

[32] D. Marsh, Handbook of Lipid Bilayers (CRC Press, Boca Raton, 2013).

[33] A. C. Woodka, P. D. Butler, L. Porcar, B. Farago, and M. Nagao, Phys. Rev. Lett. 109, 058102 (2012). 